\title{
ARTICLE
}

\section{Advances in fission-track detection and analysis for nuclear forensics and safeguards investigations}

\author{
Itzhak Halevy $^{\mathrm{a}, \mathrm{b} *}$, Uri Admon ${ }^{\mathrm{a}}$, Ernesto Chinea-Cano ${ }^{\mathrm{c}}$, Aryeh M. Weiss ${ }^{\mathrm{d}}$, Naida Dzigal ${ }^{\mathrm{c}}$, Eli Boblil ${ }^{\mathrm{a}}$, Michal Dagan ${ }^{\mathrm{a}}$, \\ Itzhak Orion $^{\mathrm{b}}$ and Roman Radus ${ }^{\mathrm{b}}$ \\ ${ }^{a}$ Department of Physics, IAEC-NRCN, Beer-Sheva 84190 Israel; ${ }^{b}$ Department of Nuclear Engineering, Ben-Gurion University - \\ Negev, Beer-Sheva 849900 Israel; ' $S G A S$ Laboratories, IAEA-Seibersdorf, A-1400, Vienna, Austria; ${ }^{d}$ Faculty of Engineering, Bar \\ Ilan University, Ramat Gan 52900 Israel
}

\begin{abstract}
A major task in nuclear forensics and safeguards investigations is the detection and analysis of small particles containing fissile isotopes that may be present at low concentration within particulate environmental samples. These radioactive particles-of-interest (POIs) are usually in the micro-metric size range, and intermixed within large populations of other particles, such as dust, soil, or industrial exhaust pollutants. A commonly used method for the detection of particles of single POIs containing fissile isotopes is to embed a dispersion of particles within a thin polymer layer ("catcher"), attach a "detector" sheet (for example, LEXAN ${ }^{\circledR}$ ) to the catcher, irradiate this structure with thermal neutrons, and then separate the two and chemically etch the detector to "develop" fission track (FT) clusters. The resulting FT clusters are visible under light microscopy, and indicate the presence of ${ }^{235} \mathrm{U}$ or other fissile isotopes within the POI. Measuring the coordinates of the FT cluster in the detector image enables the POI to be retrieved from the corresponding location in the catcher for further analysis. The main challenge in this procedure is imaging a large area detector (typically 100-400 $\mathrm{mm}^{2}$ ), at a resolution $\leq 1 \mu \mathrm{m}$, and automatically scan and locate the FT clusters while rejecting artifacts. This paper reports a novel approach which enhances the ability of the Particle Analyst to accurately identify and locate FT clusters.
\end{abstract}

\section{Keywords: fission tracks; nuclear forensics; safeguards investigations}

\section{Introduction}

This paper focuses on the application of automated quantitative microscopy to fission track (FT) cluster detection and analysis. A FT is a microscopic-scale radiation damaged site on a Solid State Nuclear Track Detector (SSNTD) that is composed of a radiant array of individual fission fragments tracks. These tracks are developed and made visible by chemical etching of the SSNTD. FT clusters arise from individual particles, and form a stochastically round shape that can be imaged with a light microscope. The SSNTD is often a polycarbonate such as LEXAN $^{\circledR}$, but other translucent polymer materials can be used. This task is part of an on-going collaboration between the International Atomic Energy Agency (IAEA) and the Israel Atomic Energy Commission (IAEC) [1], whose goal is to enhance the toolbox available to Particle Analysts in nuclear forensics and safeguards investigations.

In a previous paper [2], the basic tools developed for imaging large area detectors at sub-micron resolution, and an image processing workflow capable of detecting

*Corresponding author. Email: halevy.itzhak.dr@gmail.com and identifying FT clusters within the large images produced, were presented.

This paper extends our previous work by demonstrating the ability to compare FT clusters produced by the same POIs under different irradiation times at a given neutron flux; to compare different detectors (that may also be subjected to varying etching conditions) for the same catcher and identical irradiation conditions ; and to produce doublet sets (with varying relative intensities) of FT clusters on the same detector, by slightly shifting the detector with respect to the catcher at a chosen stage of the irradiation. The above abilities may prove useful in optimizing the FT analysis methodology, particularly in evaluating detector performance, and the refinement of irradiation and etching conditions, imaging, and image analysis.

\section{Materials and methods}

\subsection{Sample specifications and preparation}

ApproximateIy $10 \mathrm{mg}$ of IAEA-314 Stream Sediment reference material [3] was dissolved in $0.75 \mathrm{~mL}$ of heptane, and then added to a solution of $0.5 \mathrm{~g}$ of 


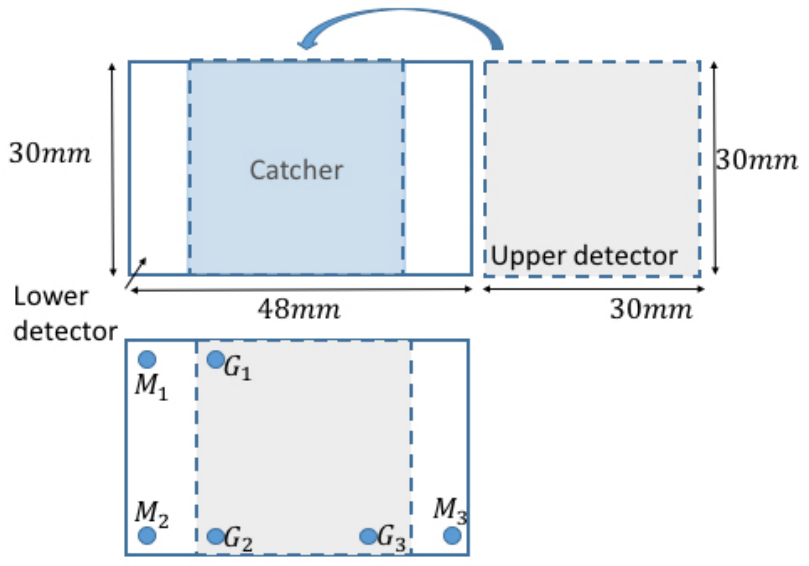

(a)

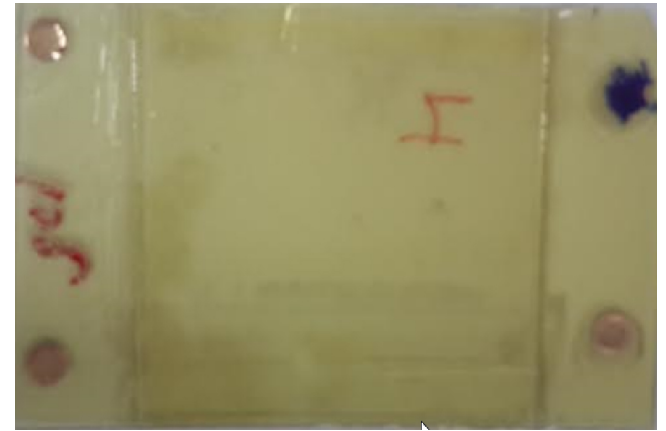

(b)

Figure 1. (a) The detector/catcher/detector "sandwich" assembly. (b) Picture of sandwich assembly. The larger lower detector is behind the smaller (upper) detector. The three fiducial markers of the lower detector are visible, while those of the upper detector have not yet been placed. The catcher that is between the two detectors is not visible.

LEXAN $^{\circledR}$ dissolved in $2 \mathrm{~mL}$ of a $1: 2$ mixture of dichloromethane and dichloroethane. This solution was mixed ultrasonically for $5 \mathrm{~min}$, and then poured onto a glass slide $(25 \mathrm{~mm} \times 25 \mathrm{~mm})$ and allowed to dry for 24 h. The resulting "catcher" was about $15 \mu \mathrm{m}$ thick.

Once fully dried, the catcher was removed from the glass slide, centered on a $48 \times 30 \mathrm{~mm}^{2}$ LEXAN $^{\circledR}$ sheet (the "lower" detector, Figure 1), and glued along its edges to the lower detector. A second LEXAN ${ }^{\circledR}$ detector, $30 \times 30 \mathrm{~mm}^{2}$ (the "upper" detector), was then placed above the catcher and attached with adhesive tape to the lower detector. The catcher was thus sandwiched between the upper and lower detectors (Figure 1).

The catcher/detectors sandwich was inserted into a pneumatic capsule (the so-called "rabbit") and irradiated in a flux of thermal neutrons $\left(10^{12} \mathrm{n} / \mathrm{cm}^{2} \mathrm{~s}\right)$ in the swimming-pool reactor at the Soreq Nuclear Research Center. Two experiments were carried out, each with a total irradiation time of $30 \mathrm{~min}$. In the first, the irradiation was un-interrupted. In the second, the irradiation was split into two cycles $(20+10 \mathrm{~min})$, and between them the upper detector was shifted slightly (about $100 \mu \mathrm{m}$ ) with respect to the catcher and lower detector.

Following an activation cooling off period of three days, three fiducial markers ( $3 \mathrm{~mm}$ copper EM grids) were glued to each detector (Figure 1). The fiducial markers form a joint coordinate system for transformation and navigation between the two detectors (with the catcher attached to the lower detector) [4]. Once the fiducial markers were placed, and their relative coordinates measured, the detectors were separated from the detector-catcher-detector sandwich and chemically etched for $13 \mathrm{~min}$ in a $6 \mathrm{~N} \mathrm{NaOH}$ solution, at $70{ }^{\circ} \mathrm{C}$. The etching serves to "develop" the FT clusters, making them visible under light microscopy. This study used the upper detector.

\subsection{Microscopy}

Imaging was done on a fully motorized Nikon TE2000E inverted microscope (Nikon Instruments Inc., Tokyo, Japan), equipped with the PFS (Perfect Focus System) focus control system and a Märzhäuser L-STEP $\mathrm{XY}$-motorized stage. The microscope was controlled with Nikon NIS-Elements software. Large area images were acquired using a $4 \times / \mathrm{NA}=0.2$ objective and a Nikon DS-Qi2 16 MP (4908x3264) CMOS imager, coupled to the scope with a $2.5 \times$ adapter. The pixel pitch was 0.73 $\mu \mathrm{m} /$ pixel. The FOV of this imager was about $3.58 \mathrm{~mm} \times$ $2.38 \mathrm{~mm}$. Large areas were acquired by tiling many fields, with the necessary overlap (typically $10 \%$ ). The NIS Elements software autofocus was used in order to maintain focus over the large areas (over $2 \times 2 \mathrm{~cm}$ ) that were acquired. A background illumination image was also acquired, in order to correct for illumination non-uniformity, prior to stitching. However, we found that a software generated background image provided a better illumination correction estimate than the measured illumination image. This background image was generated by averaging many tiles that were acquired, and then blurring that average with a Gaussian blurring filter set to a radius of 100 pixels.

\subsection{Image processing}

Image processing was done using the Fiji distribution of ImageJ $[5,6]$. The algorithms used in this study were implemented using either the ImageJ macro language or the ImageJ Python scripting engine. The images were corrected for nonuniform illumination and fixed instrumental noise (e.g. dust on the imager surface) by dividing each tile by the background illumination image mentioned above, and then restoring the mean value to the result. The background corrected tiles were then stitched using the ImageJ Grid/Collection stitching plugin [7]. The MaxEntropy auto-thresholding method of ImageJ was used to segment the illumination corrected images. 


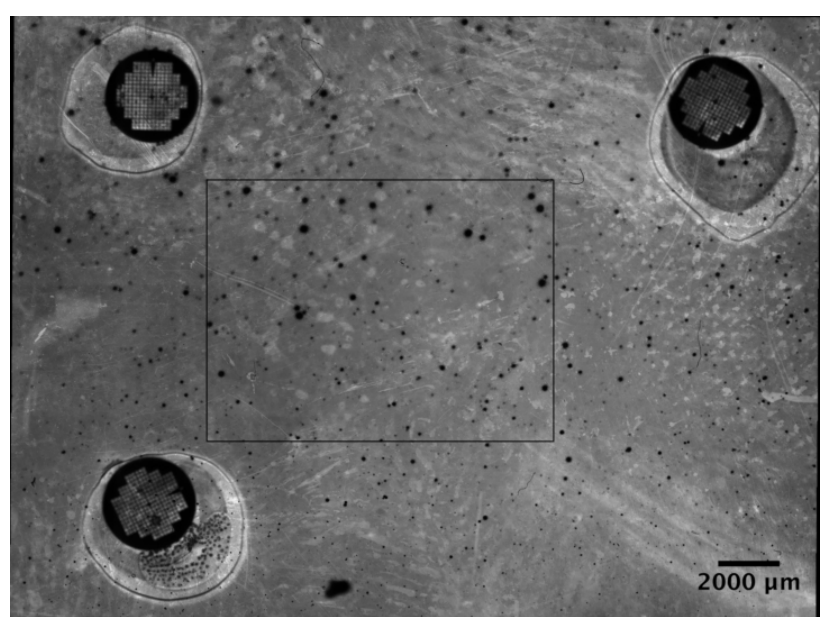

(a)

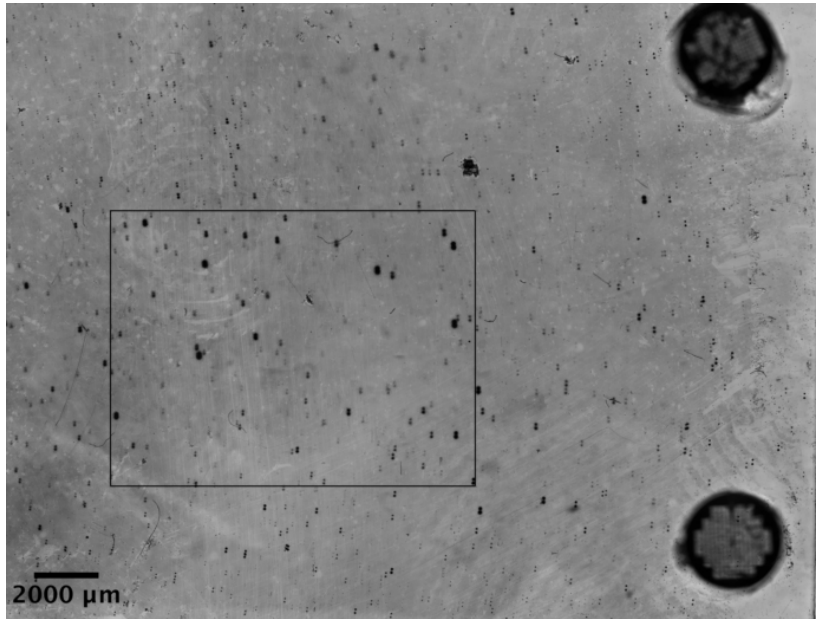

(b)

Figure 2. (a) Image of detector irradiated for $30 \mathrm{~min}$. (b) A second detector, irradiated for $20 \mathrm{~min}$, then shifted (see text), and finally irradiated for an additional 10 min under the same neutron flux.

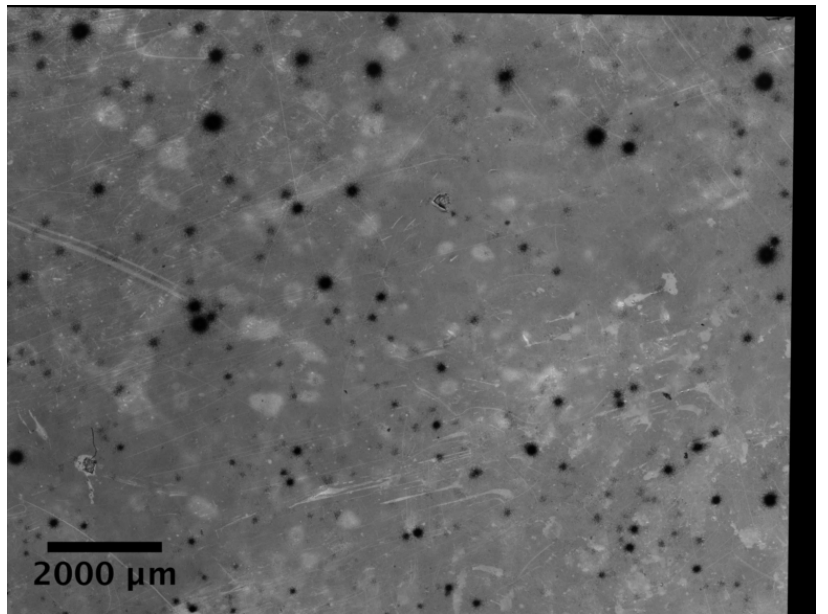

(a)

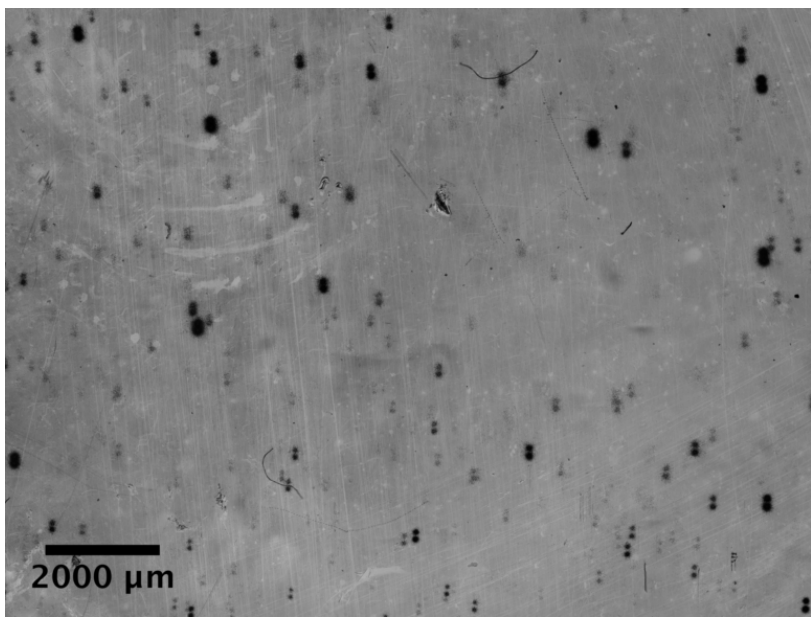

(b)

Figure 3. (a) Zoom-in to the area marked in Figure 2a (b) Zoom-in to the area marked in Figure 2b. The Image in (a) has been registered to the image in (b), so that the FT clusters overlap when the two images are superimposed.

\section{Results}

Figure 2 shows large area scans of two detectors that were irradiated using the same catcher, and therefore the same particle distribution. Figure $2 \mathrm{a}$ is an image of a detector that was irradiated for $30 \mathrm{~min}$, while Figure $2 \mathrm{~b}$ is an image of a detector that was irradiated for $20 \mathrm{~min}$, shifted slightly as described in the methods section, and finally irradiated for an additional $10 \mathrm{~min}$. As expected, the FT cluster size increases with increasing irradiation times.

Figure 3 shows images of the areas from within the rectangles marked in Figure 2. The image in Figure 3a has been shifted slightly in order to align its FT clusters with those in Figure $3 b$, so that if the images were overlaid, the FT clusters would overlap. The alignment was computed automatically with the StackReg plugin [8], using a rigid body transformation.

Figure 4 shows two prominent FT clusters that appear in the upper right corner of the images in Figure
3. The MaxEntropy automatic threshold method available in ImageJ was used to detect the FT clusters, and then the Ellipse-Split plugin [9] was used to fit ellipses to the segmented objects. The FT in Figure 4a were not divided by the plugin into two ellipses, because in this image the FT clusters are single round clusters. However, in Figure $4 \mathrm{~b}$, each cluster is really a pair of clusters, offset from each other by about 35-40 pixels in the vertical (Y) direction. The Ellipse-Split algorithm automatically picks this up and fits two overlapping ellipses to each FT cluster doublet. Careful examination of Figure $3 \mathrm{~b}$ reveals that all of the FT clusters are doublets. Segmentation and Ellipse-Split of these objects produce doublets whose separation is a measure of the distance that the detector shifted between exposures. This characteristic of the FT clusters can be exploited to distinguish between true FT clusters and artifacts such as dirt on the detector. 


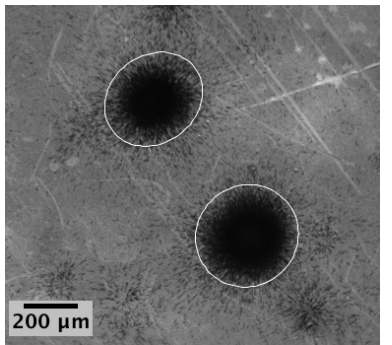

(a)

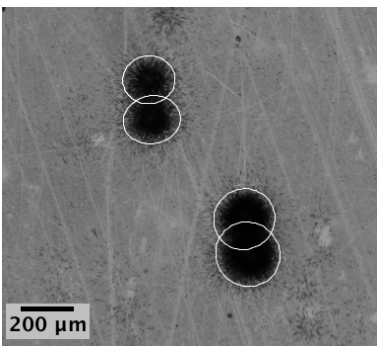

(b)
Figure 4. (a) Zoom-in to FT in the upper right of Figure 3a;(b) Zoom-in to FT in the upper right of Figure 3b. The FT cluster were segmented, and then the Ellipse-Fit plug-in was used to find the best fit ellipses to these objects. These are shown in white on the images.

\section{Discussion and conclusions}

When imaging large area SSNTDs, one is faced with two problems: (1) the size of the images that are produced and (2) the need to automatically locate the FT clusters, which may be sparse and masked by artifacts that produce many false positives. In our previous work [2], it was demonstrated that image size can be kept below a few gigabytes per detector, while imaging at sufficiently high optical resolution to distinguish FT from artifacts.

This paper demonstrates two ideas that have the potential to significantly reduce the resolution required to distinguish true FT clusters from artifacts. They are:

1. multiple exposure of the detector, with a slight shift between exposures, resulting in characteristic FT doublet patterns.

2. exposure of multiple detectors using the same catcher. In this case, the true FT will appear on all of the detectors, while the artifacts will be uncorrelated. We note that the lower detector gives us a duplicate "for free".

In addition, FT clusters created with different exposure times are compared. The longest irradiation times result in large FT clusters that can be many tens and even hundreds of micrometers in extent. Such large FT clusters can be easily located. When this consideration is combined with the ideas of multiple exposures of a single detector, and/or exposure of two detectors using a single fixed distribution of particles (i.e., in the same catcher), reliable FT detection together with rapid imaging of the detectors should be easily accomplished. In this connection, it is noteworthy that the irradiation doses used in FT analyses cause only negligible degradation of the fissile isotope concentration in the POIs. Therefore, multiple exposure experiments using the same catcher are feasible.

\section{References}

[1] U. Admon, E. Chinea-Cano, N. Dzigal, I. Halevy, E. Boblil, T. Elkayam, A. Weiss and S. Vogt. Advancements in particle analysis procedures and their application in characterizing reference materials for safeguards, In Proc. Symp. on International Safeguards: Linking Strategy, Imple-mentation and People, IAEA, Vienna (2014), p. 227. URL: https://www.iaea.org/safeguards/ symposium/2014/home/eproceedings/sg2014_epro ceedings_online.pdf.

[2] A.M. Weiss, I. Halevy, N. Dzigal, E. Chinea-Cano and U. Admon, Fission track detection using automated microscopy, Journal of Nuclear Engineering and Radiation Science 3 (2017), pp. 030910-030910-7.

[3] V. Strachnov, V. Valkovic, R. Zeisler and R. Dekner, Intercomparison study iaea-314 on the determination of $\mathrm{u}$, th and ra-226 in stream sediment, Technical report, Vienna, Austria (1991). URL: https://nucleus.iaea.org/rpst/Documents/rs iaea-314.pdf.

[4] U. Admon, Single particle handling and analysis, In D. Oughton and V. Kashparov, eds., Radioactive Particles in the Environment, Springer, Dordrecht, The Netherlands (2009), pp. 15-55.

[5] C.A. Schneider, W.S. Rasband and K.W. Eliceiri, NIH Image to ImageJ: 25 years of image analysis, Nature Methods 9 (2012), pp. 671-675.

[6] J. Schindelin, I. Arganda-Carreras and E. Frise, Fiji: an open-source platform for biological-image analysis, Nature Methods 9 (2012), pp. 676-682.

[7] S. Preibisch, S. Saalfeld and P. Tomancak, Globally optimal stitching of tiled 3d microscopic image acquisitions, Bioinformatics 25 (2009), pp. 1463-1465. URL: http://bioinformatics.oxfordjour nals.org/content/25/11/1463.abstract.

[8] P. Thévenaz, U. Ruttimann and M. Unser, A pyramid approach to subpixel registration based on intensity, IEEE Transactions on Image Processing 7 (1998), pp. 27-41.

[9] T. Wagner, Ellipse Split (2017) (accessed 31 July 2017). URL: https://zenodo.org/record/834339. 